\title{
Взаимодействие таммовского плазмона и экситона в органическом материале в режиме сильной связи
}

\author{
(C) К.М. Морозов ${ }^{1}$, А.В. Белоновский ${ }^{1}$, К.А. Иванов ${ }^{2}$, Е.И. Гиршова ${ }^{1,3}$, М.А. Калитеевский ${ }^{1,2,3,9}$ \\ ${ }^{1}$ Санкт-Петербургский Академический университет, \\ 194021 Санкт-Петербург, Россия \\ 2 Университет ИТМО, \\ 197101 Санкт-Петербург, Россия \\ ${ }^{3}$ Физико-технический институт им. А.Ф. Иоффре Российской академии наук, \\ 194021 Санкт-Петербург, Россия \\ ฯ E-mail: Kalit@mail.ioffe.ru
}

Поступила в Редакцию 24 апреля 2019 г.

В окончательной редакции 29 апреля 2019 г.

Принята к публикации 29 апреля 2019 г.

\begin{abstract}
Проведено теоретическое исследование взаимодействия таммовского плазмона с экситоном в органическом материале в режиме сильной связи. Структура представляла собой брэгговский отражатель из 5 пар слоев оксида кремния и оксида тантала, органического светоизлучающего слоя материала 4'-Bis $(N$-carbazolyl)-1,1'-biphenyl и слоя серебра. Показано, что в такой структуре имеет место расщепление поляритонных мод (расщепление Раби) величиной $>400$ мэВ, что может сопровождаться увеличением ширины полосы люминесценции до 700 мэВ.
\end{abstract}

Ключевые слова: таммовский плазмон, органический светодиод, экситон, режим сильной связи.

DOI: $10.21883 /$ FTP.2019.10.48288.34

\section{1. Введение}

Перспективные источники [1-4] и детекторы света $[5,6]$ на основе таммовских плазмонов (состояний электромагнитного поля, локализованных на границе брэгговского отражателя и металла [7]) интенсивно исследуются в последнее время. В некоторых органических светоизлучающих материалах экситоны обладают очень высокой силой осциллятора, что приводит к взаимодействию экситона в органическом материале и собственной оптической моды микрорезонатора в режиме сильной связи [8], причем величина расщепления поляритонных мод (расщепление Раби) может достигать нескольких сотен мэВ. В микрорезонаторах с органическими материалами люминесценция в режиме сильной связи наблюдается только из нижней поляритонной моды. В структурах с таммовскими плазмонами и полупроводниковыми квантовыми ямами люминесценция экспериментально наблюдалась и для верхней поляритонной ветки [9].

Недавно было продемонстрировано увеличение скорости спонтанной эмиссии в структурах с таммовским плазмоном с активной областью из квантовых точек [10], таким образом, структуры с таммовскими плазмонами могут использоваться для высокоэффективных резонансных светодиодов.

Данная работа посвящена теоретическому исследованию взаимодействия таммовского плазмона и экситона в органическом материале с целью определения дизайна структуры, обеспечивающей люминесценцию как из нижней, так и из верхней поляритонной моды.

\section{2. Результаты и обсуждение}

Была рассмотрена структура с таммовским плазмоном, состоящая из распределенного брэгговского отражателя (5 пар $\mathrm{Ta}_{2} \mathrm{O}_{5} / \mathrm{SiO}_{2}$ слоев на подложке $\mathrm{SiO}_{2}$ с толщинами 39 и 66 нм соответственно), активного органического слоя материала CBP $\left(4,4^{\prime}\right.$-Bis $(N$-carbazolyl)1,1'-biphenyl) толщиной 26 нм и слоя серебра толщиной 50 нм. Схема структуры показана на рис. 1,a. Рассчитанный спектр отражения при нормальном падении света от $\mathrm{Ta}_{2} \mathrm{O}_{5} / \mathrm{SiO}_{2}$ брэгговского отражателя представлен на рис. $1, b$ пунктирной линией. Сплошной линией на рис. $1, b$ показан спектр отражения при нормальном падении от структуры с таммовским плазмоном, показанной на рис. 1,a. Провал в спектре отражения на частоте $\omega=3.21$ эВ соответствует таммовскому плазмону, профиль электрического поля таммовского плазмона показан на рис. 1,c. Электрическое поле локализовано вблизи границы слоя серебра (светло-серая область) и СВР (темно-серая область), в котором и находится максимум электрического поля.

На рис. 2 показана спектральная зависимость действительной и мнимой частей показателя преломления СВР в экситонной области спектра, описываемая выражением

$$
\varepsilon_{\mathrm{CBP}}(\omega)=\varepsilon_{b}+\frac{\omega_{\mathrm{LT}}}{\omega_{\mathrm{exc}}-\omega-i \gamma_{\mathrm{exc}}},
$$

где $\varepsilon_{b}-$ фоновая диэлектрическая проницаемость, $\omega_{\mathrm{LT}}$ - продольно-поперечное расщепление, $\omega_{\mathrm{exc}}-$ резонансная частота и $\gamma_{\text {exc }}$ - нерадиационное затухания экситона $\left(150\right.$ мэВ), значения которых равны $\varepsilon_{b}=3.2$, $\omega_{\mathrm{LT}}=130$ мэВ [11]. 

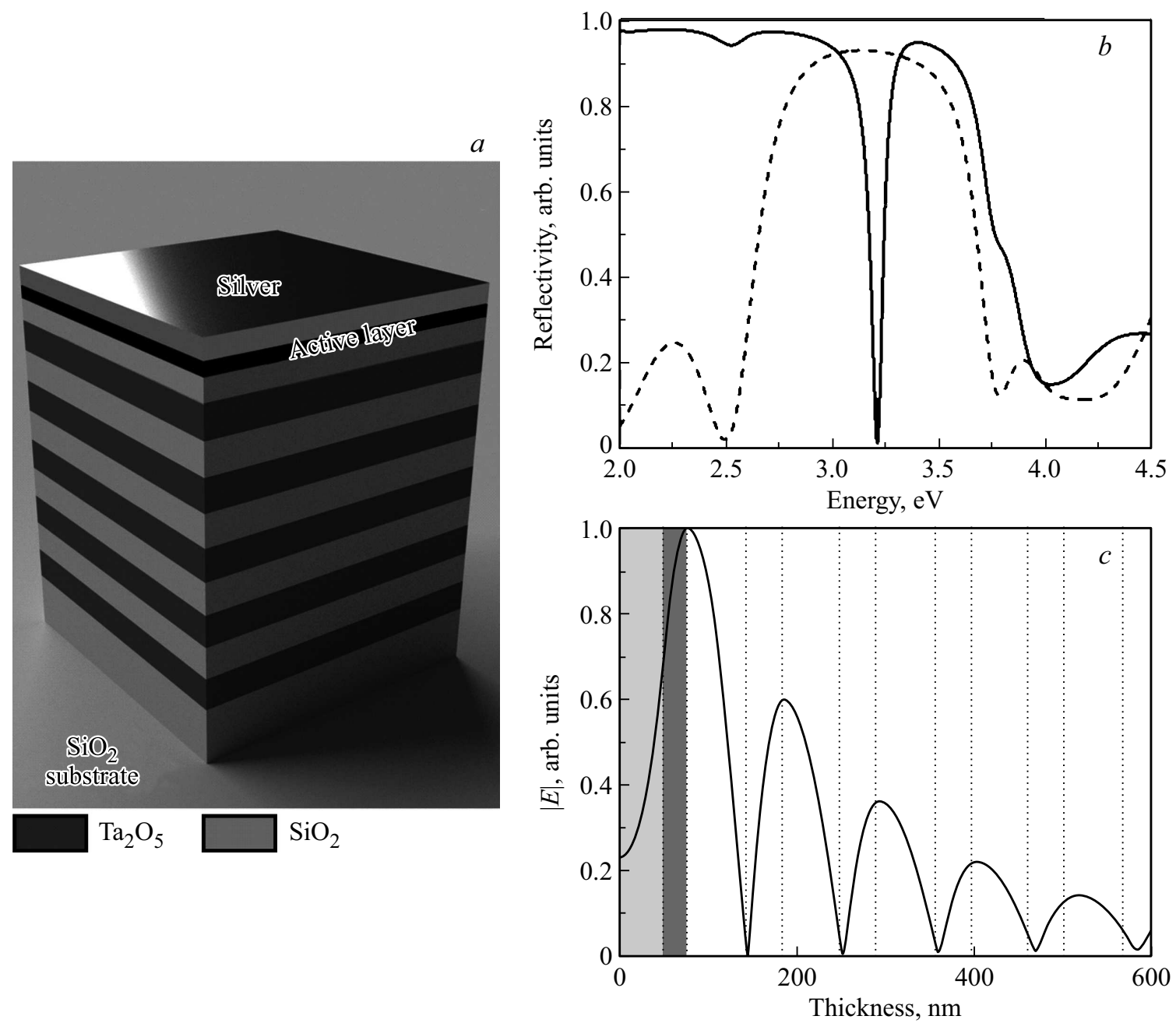

Рис. 1. $a-$ схема структуры с таммовским плазмоном с органической активной областью. $b-$ спектры отражения структуры с таммовским плазмоном (сплошная линия) и распределенного брэгговского отражателя (пунктирная линия) при нормальном падении. $c$ - распределение модуля электрического поля в случае ТЕ-поляризации. Точечные линии демонстрируют положения интерфейсов между слоями. Серая и темно-серая области соответствуют слою серебра и активной области СВР соответственно.

Можно видеть, что экситон в СВР имеет частоту $\omega_{\mathrm{exc}}=3.52$ эВ, при этом линия излучения сдвинута относительно экситона в сторону низких энергий на $\sim 300$ мэВ.

Используя модель связанных осцилляторов, можно получить частоты поляритонных мод, которые задаются выражением

$$
\omega_{\mathrm{UP}, \mathrm{LP}}=\frac{\omega_{\mathrm{exc}}+\omega_{\mathrm{TP}}}{2}-\frac{i\left(\gamma_{\mathrm{exc}}+\gamma_{\mathrm{TP}}\right)}{2} \pm \frac{\Omega_{\mathrm{Rabi}}}{2},
$$

где

$$
\Omega_{\mathrm{Rabi}}=2 \sqrt{V^{2}+\frac{1}{4}\left(\omega_{\mathrm{exc}}-\omega_{\mathrm{TP}}-i\left(\gamma_{\mathrm{exc}}-\gamma_{\mathrm{TP}}\right)\right)^{2}}
$$

- расщепление Раби, $V=\sqrt{\omega_{\text {еxс }} \omega_{\mathrm{LT}}}-$ константа связи таммовского плазмона с экситоном, $\gamma_{\text {TP }}$ - затухание моды таммовского плазмона [12].

Рис. 3 иллюстрирует возникновение сильной связи при взаимодействии экситона с таммовским плаз-

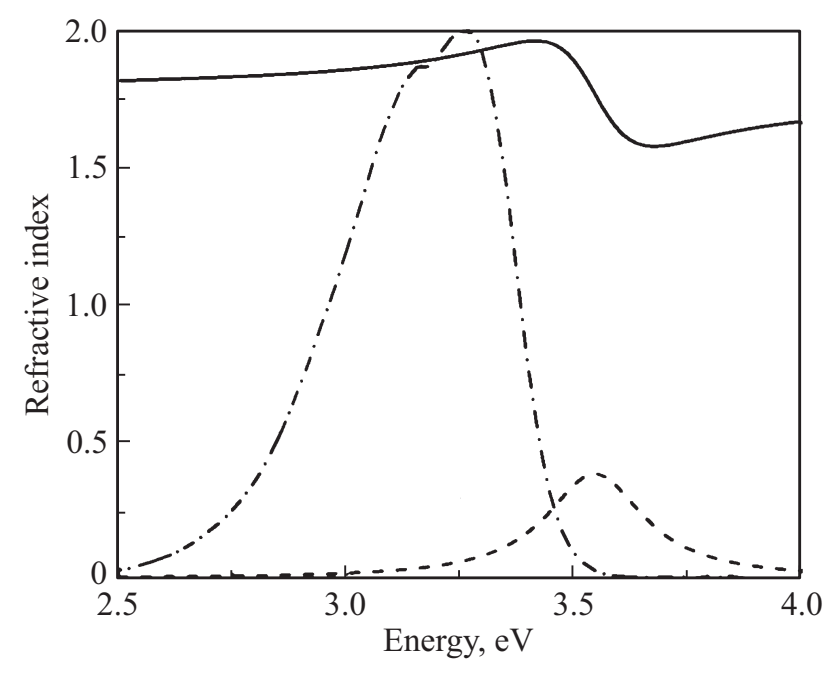

Рис. 2. Действительная (сплошная линия) и мнимая (пунктирная линия) части показателя преломления органического материала CBP (4,4'-Bis( $N$-carbazolyl)-1,1'-biphenyl). Штрихпунктирная линия показывает спектр излучения материала. 

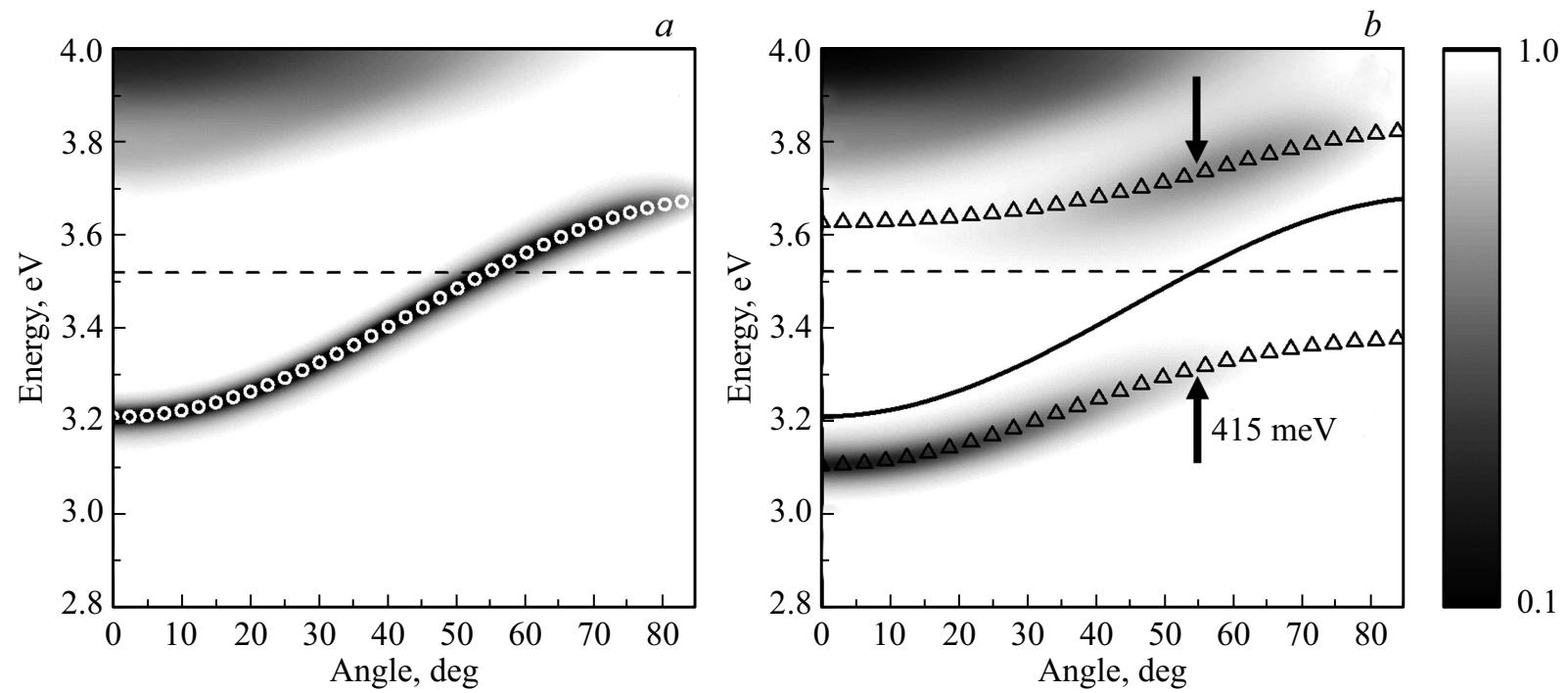

Рис. 3. $a$ - рассчитанная зависимость коэффициента отражения от энергии и угла для структуры с таммовским плазмоном при отсутствии экситонного вклада в диэлектрической проницаемости органического материала в случае ТЕ-поляризации. Кружки показывают дисперсию таммовского плазмона. $b$ - рассчитанная зависимость коэффициента отражения от энергии и угла для структуры с таммовским плазмоном в режиме сильной связи. Треугольники показывают дисперсии верхней и нижней поляритонных ветвей. Сплошная линия - дисперсия таммовского плазмона. Пунктирная линия показывает энергию экситонного резонанса.
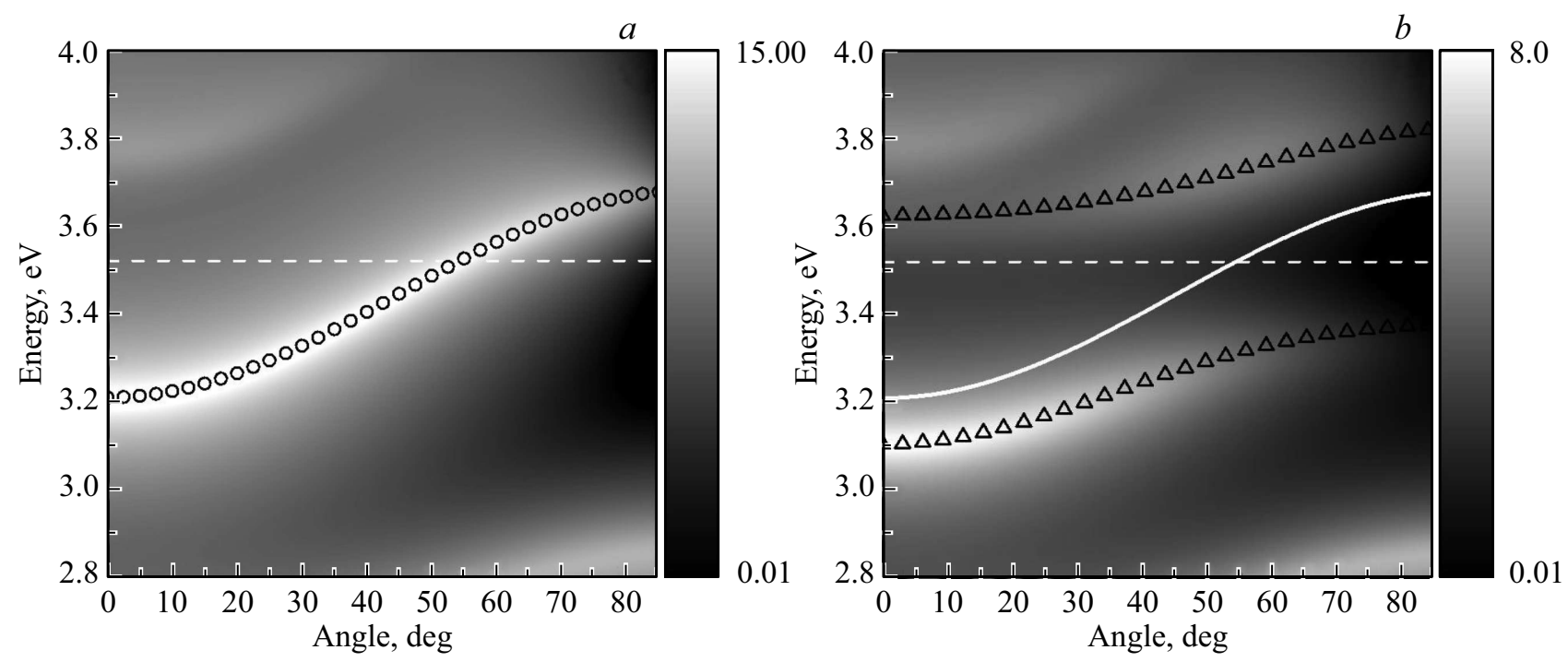

Рис. 4. $a$ - рассчитанная зависимость модового коэффициента Парселла от энергии и угла для структуры с таммовским плазмоном при отсутствии экситонного вклада в диэлектрической проницаемости органического материала в случае ТЕполяризации. Кружки показывают дисперсию таммовского плазмона. $b-$ рассчитанная зависимость модового коэффициента Парселла от энергии и угла для структуры с таммовским плазмоном в режиме сильной связи. Треугольники показывают дисперсии верхней и нижней поляритонных ветвей. Сплошная линия - дисперсия таммовского плазмона. Пунктирная линия показывает энергию экситонного резонанса.

моном. На рис. 3, а показана зависимость коэффициента отражения от рассматриваемой структуры при отсутствии в спектральной зависимости диэлектрической проницаемости СВР (при $\left.\omega_{\mathrm{LT}}=0\right)$. Можно видеть провал, соответствующий таммовскому плазмону. При наличии экситонной особенности в СВР, в спектрах отражения можно видеть два провала (рис. 3,b), соответствующих поляритонным модам, и дисперсионные зависимости верхней и нижней поляритонных мод имеют характер антипересечения с расщеплением > 400 мэВ.

Вследствие эффекта Парселла вероятность спонтанной эмиссии для излучателя в микрорезонаторе может быть увеличена. В структурах с таммовским плазмо- 
ном наблюдалось увеличение вероятности спонтанной эмиссии для частот и направлений излучения, соответствующих таммовскому плазмону в режиме слабой связи [10]. Значительное увеличение вероятности спонтанной эмиссии в таких структурах объясняется высокой степенью локализации электрического поля моды в активном слое.

На рис. 4 показаны рассчитанные зависимости модального коэффициента Парселла $[10,13]$ от частоты и направления эмиссии при отсутствии экситонной особенности (рис. $4, a$ ) и при ее наличии (рис. $4, b$ ). Можно видеть, что при отсутствии экситонной особенности в области частот и направлений, соответствующих таммовскому плазмону, вероятность спонтанной эмиссии увеличена. При взаимодействии таммовского плазмона с экситоном в СВР при малых углах эмиссии имеет место усиление спонтанной эмиссии для нижней поляритонной ветки, а при углах эмиссии $>50^{\circ}$ - для верхней поляритонной ветки, такое поведение обусловлено различиями в пространственных профилях электрического поля для верхней и нижней поляритонных мод.

Процессы рассеяния неравновесных поляритонов в органических светоизлучающих веществах могут характеризоваться малой скоростью, что позволяет наблюдать люминесценцию из высокоэнергетичных областей поляритонных мод [14].

Увеличение вероятности спонтанной эмиссии для верхней и нижней поляритонных мод делает возможным люминесценцию на частотах, соответствующих как нижней, так и верхней поляритонным модам, что может существенно увеличить ширину полосы люминесценции структуры (до 700 мэВ) по сравнению с полосой люминесценции материала (450 мэВ).

\section{3. Заключение}

Был проведен теоретический анализ возможности использования эффекта сильной связи между экситоном в органическом материале и таммовским плазмоном для создания светоизлучающих структур с широкой полосой излучения. Исследуемая структура представляла собой брэгговский отражатель из слоев оксида кремния и оксида тантала на подложке оксида кремния, на который были нанесены слои органического 4,4'-Bis ( $N$-carbazolyl)$1,1^{\prime}$-biphenyl (CBP) и серебра. Были проведены расчеты коэффициента отражения и вероятности спонтанной эмиссии от частоты и направления эмиссии. Продемонстрировано, что при взаимодействии экситона в СВР и таммовского плазмона в режиме сильной связи ширина полосы люминесценции структуры существенно увеличивается по сравнению с шириной полосы люминесценции материала.

\section{Благодарности}

Авторы выражают благодарность Российскому научному фонду (проект № 16-12-10503).

\section{Конфликт интересов}

Авторы заявляют, что у них нет конфликта интересов.

\section{Список литературы}

[1] R. Brückner, A.A. Zakhidov, R. Scholz, M. Sudzius, S.I. Hintschich, H. Fröb, V.G. Lyssenko, K. Leo. Nature Photonics, 6 (5), 322 (2012).

[2] C. Symonds, G. Lheureux, J.P. Hugonin, J.J. Greffet, J. Laverdant, G. Brucoli, A. Lemaitre, P. Senellart, J. Bellessa. Nano Lett., 13 (7), 3179 (2013).

[3] A.R. Gubaydullin, C. Symonds, J.-M. Benoit, L. Ferrier, T. Benyattou, C. Jamois, A. Lemaitre, P. Senellart, M.A. Kaliteevski, J. Bellessa. Appl. Phys. Lett., 111 (26), 261103 (2017).

[4] R. Bikbaev, S. Vetrov, I. Timofeev. Photonics, 5 (3), 22 (2018).

[5] S.-G. Huang, K.-P. Chen, S.-C. Jeng. Opt. Mater. Express, 7 (4), 1267 (2017).

[6] S.V. Boriskina, Y. Tsurimaki. J. Phys. Condens. Matter, 30 (22), 224003 (2018).

[7] M. Kaliteevski, I. Iorsh, S. Brand, R.A. Abram, J.M. Chamberlain, A.V. Kavokin, I.A. Shelykh. Phys. Rev. B, 76 (16), 165415 (2007).

[8] D. Ballarini, M. De Giorgi, S. Gambino, G. Lerario, M. Mazzeo, A. Genco, G. Accorsi, C. Giansante, S. Colella, S. D’Agostino, P. Cazzato, D. Sanvitto, G. Gigli. Adv. Optical Mater., 2, 1076 (2014).

[9] C. Symonds, A. Lemaitre, E. Homeyer, J.C. Plenet, J. Bellessa. Appl. Phys. Lett., 95 (15), 151114 (2009).

[10] A.R. Gubaydullin, C. Symonds, J. Bellessa, K.A. Ivanov, E.D. Kolykhalova, M.E. Sasin, A. Lemaitre, P. Senellart, G. Pozina, M.A. Kaliteevski. Sci. Rep., 7, 9014 (2017).

[11] B. Deveaud. The Physics of Semiconductor Microcavities: From Fundamentals to Nanoscale Devices (Wiley-VCH Verlag GmbH \& Co., 2007).

[12] E.L. Ivchenko, M.A. Kaliteevski, A.V. Kavokin, A.I. Nesvizhskii. J. Opt. Soc. Am. B, 13 (5), 1061 (1996).

[13] K.M. Morozov, A.R. Gubaydullin, K.A. Ivanov, G. Pozina, M.A. Kaliteevski. J. Phys. Condens. Matter, 30, 435304 (2018).

[14] D.M. Coles, P. Michetti, C. Clark, W.C. Tsoi, A.M. Adawi, J.-S. Kim, D.G. Lidzey. Adv. Funct. Mater., 21, 3691 (2011).

Редактор А.Н. Смирнов 
Interaction of Tamm plasmon and exciton in organic material in the strong coupling regime

K.M. Morozov ${ }^{1}$, A.V. Belonovskii ${ }^{1}$, K.A. Ivanov ${ }^{2}$, E.I. Girshova ${ }^{1}$, M.A. Kaliteevski ${ }^{1,2,3}$

${ }^{1}$ St. Petersburg Academic University, 194021 St. Petersburg, Russia

2 ITMO University,

197101 St. Petersburg, Russia

3 loffe Institute,

194021 St. Petersburg, Russia

Abstract Theoretical analysis of the interaction between Tamm plasmon and exciton in organic material has been carried out. The structure is 5 pair silicon oxide and tantalum oxide Bragg reflector, $4^{\prime}$-Bis $\left(N\right.$-carbazolyl)-1, $1^{\prime}$-biphenyl (CBP) organic material layer and a silver layer. In the considered structure splitting of polaritonic modes (Rabi splitting) of $400 \mathrm{meV}$ order was demonstrated. Splitting may be accompanied with an increase in luminescence bandwidth up to $700 \mathrm{meV}$. 